\title{
Stark broadening of Balmer lines with low and moderate quantum number in dense divertor plasmas
}

\author{
J. Rosato, ${ }^{1}$ N. Kieu, ${ }^{1}$ M. Meireni, ${ }^{1}$ R. Sheeba, ${ }^{1}$ M. Koubiti, ${ }^{1}$ Y. Marandet, ${ }^{1}$ R. Stamm, ${ }^{1}$ K. \\ Verhaegh, ${ }^{2,3}$ B. Duval, ${ }^{2}$ the TCV team|t and the MST1 team|" \\ ${ }^{1}$ PIIM, UMR 7345 Aix-Marseille Université / CNRS, Centre de St-Jérôme, Case 232, F-13397 Marseille Cedex 20, \\ France \\ ${ }^{2}$ Ecole Polytechnique Fédérale de Lausanne (EPFL), SPC, Lausanne, CH \\ ${ }^{3}$ York Plasma Institute, University of York, York, UK
}

Correspondence: J. Rosato. Email: joel.rosato@univ-amu.fr

Received ; Revised ; Accepted

\section{Summary}

A computer simulation technique is applied to the modeling of Balmer line shapes in dense divertor conditions. The spectral profile of lines with a high principal quantum number $n$ is sensitive to Stark broadening and can be used as a density diagnostic. In contrast, an analysis of the shape of low or moderate $n$ lines such as $\mathrm{D} \alpha(n=3), \mathrm{D} \beta$ $(n=4)$ and $\mathrm{D} \gamma(n=5)$ is more intricate because the Stark effect is weaker and can compete with the thermal Doppler broadening. We examine this issue and address the relative contribution of the Stark and Doppler effects on the first Balmer lines. Analyses of experimental spectra are performed.

Keywords: Spectroscopy, Stark effect, plasma diagnostic

\section{Introduction}

Passive spectroscopy provides a non-intrusive diagnostic of tokamak edge plasmas: an analysis of the shape and the intensity of atomic spectral lines yields local values of the plasma parameters $\left(N_{e}, T_{e}\right.$ etc.), provided a suitable model can be found. A specific issue that requires careful examination is the broadening of the hydrogen isotope lines observed in the edge and in the divertor of tokamaks. In detached regime, the plasma located near the divertor target plates contains a large proportion of neutrals in an excited state, with a principal quantum number $n$ possibly larger than 10. The corresponding dipole moment is large enough so that the atomic energy levels are sensitive to the plasma's microscopic electric field (Stark effect). Accordingly, the emission lines observed in passive spectroscopy can be used as a probe of the electron density; recent analyses have been performed on spectra observed in the

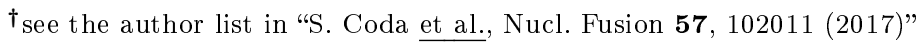

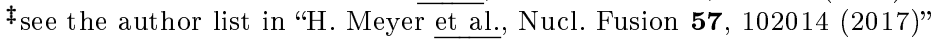


divertor of ASDEX-Upgrade [1], JET 2], and TCV [3. An issue that remains unclear concerns the validity of using a pure Stark broadening model, i.e. not accounting for other line broadening mechanisms, on Balmer lines with a low or moderate quantum number such as $\mathrm{D} \alpha$ and $\mathrm{D} \beta$ ( $n=3 \rightarrow 2$ and $4 \rightarrow 2$ transitions). In contrast to the high- $n$ lines that are more strongly affected by Stark broadening, the first lines in the Balmer series are weakly sensitive to the plasma microfield, so any thermal Doppler broadening can become significant; this property has already been used for probing the atomic velocity distribution function at the edge 4 5. In this work, we examine the relative contribution of the Stark and Doppler broadenings to the shape of the first Balmer lines. Our calculations employ a computer simulation technique, which has been designed for the description of Stark broadening in tokamak edge plasma conditions 6. The formalism and the method are presented in Sec. 2 and calculations are reported in Sec. 3. An analysis of an experimental spectrum observed in the divertor of TCV is performed in Sec. 4 .

\section{Stark broadening modeling}

The formalism used in Stark line shape modeling involves atomic operators and related quantities in the framework of quantum mechanics. Here, we give a summary of formulas; further details can be found in textbooks, e.g. 7 . Consider an atom immersed in a plasma and emitting a photon, the spectrum is given by the Fourier transform of the dipole autocorrelation function

$$
\begin{aligned}
& I(\omega)=\frac{1}{\pi} \operatorname{Re} \int_{0}^{\infty} d t C(t) e^{i \omega t}, \\
& C(t)=\operatorname{Tr}\left\{\vec{d}_{\perp}(0) \cdot \vec{d}_{\perp}(t) \rho\right\} .
\end{aligned}
$$

Here, the trace is performed over the atomic states and denotes a statistical average, $\rho$ is the projection of the density operator onto the atom's Hilbert space evaluated at the initial time, the brackets $\{\ldots\}$ denote an average over the perturber trajectories (classical path approximation), and $\vec{d}_{\perp}=\vec{d}-(\vec{d} \cdot \vec{n}) \vec{n}$ is the projection of the atomic dipole operator onto the polarization plane, in the Heisenberg picture $(\vec{n}=\vec{k} /|\vec{k}|$ where $\vec{k}$ is the wave vector). $\omega$ denotes the angular frequency of the emitted photon. In Eq. (2), restrictions of the dipole operator to the upper and lower levels of the transition are implied (no-quenching approximation). For calculation purposes, it is convenient to write the autocorrelation function in terms of an evolution operator $U(t)$. From the identity $\vec{d}_{\perp}(t) \equiv U^{\dagger}(t) \vec{d}_{\perp}(0) U(t)$, a calculation of the line shape requires knowledge of the matrix elements of $\vec{d}$ and an evaluation of $U(t)$. The evolution 
operator obeys the time-dependent Schrödinger equation

$$
i \hbar \frac{d U}{d t}(t)=\left[H_{0}+V(t)\right] U(t)
$$

Here, $H_{0}$ is the Hamiltonian including both the atomic energy level structure (with a non-Hermitian part accounting for natural broadening) and the Zeeman effect, and $V(t)=-\vec{d} \cdot \vec{E}(t)$ is the time-dependent Stark effect term (Schrödinger picture) resulting from the action of the microscopic electric field $\vec{E}(t)$. When this term is neglected, the Schrödinger equation has the trivial solution $U(t)=\exp \left(-i H_{0} t / \hbar\right)$, which shows, using Eqs. (2) and (1), that $I(\omega)$ reduces to a set of delta functions (or Lorentzian functions if the natural broadening is retained). The case where $\vec{E}(t)$ must be retained is more complex as there is no general exact analytical solution. Time-dependent perturbation theory yields a formal expansion (Dyson series), which is not applicable in explicit calculations because of the non-commutation of the interaction term at different times (time-ordering problem). This, in particular, concerns the microfield due to ions. Several models, based on suitable approximations, have been developed to provide an analytical expression for the line shape (e.g., the impact and static approximations; the model microfield method; etc.). For our purposes, it is convenient to have a line shape model with less restrictive approximations, made to be applicable to various plasma conditions. A good candidate is the simulation technique 8 . The purpose of this method is to numerically reproduce the motion of the charged particles in the plasma to obtain the time dependent

electric microfield $\vec{E}(t)$. Explicitly, a numerical simulation consists of: (i) the calculation of a set of realizations for the electric field; (ii) the numerical integration of the Schrödinger equation for each realization; (iii) the average of the evolution operators on a set of realizations and the Fourier transform of the autocorrelation function. In the simulations performed for this work, we use a code designed for weakly coupled magnetized plasmas, i.e.with a large number of particles in the Debye sphere. A deuterium atom immersed in pure deuterium plasma is assumed. Only the ion motion is simulated here and it provides a set of realizations for the ion electric field $\vec{E}_{i}(t)$, to be used in the Schrödinger equation. The contribution of the electrons is described using a damping term (collision operator). Technical details can be found in $[10]$.

\section{Relative contribution of Stark and Doppler broadening}

The formalism presented above models the line shape in the atomic frame of reference, i.e. without any Doppler effect. The Doppler broadening due to the thermal motion of the emitters can be retained through a convolution 
with the velocity distribution function (VDF) $f(\vec{v})$, using the following substitution

$$
I(\omega) \rightarrow \int d^{3} v f(\vec{v}) I\left(\omega-\omega_{0} \vec{v} \cdot \vec{n} / c\right)
$$

where $\omega_{0}$ is the central angular frequency of the line under consideration. The first lines in the Balmer series, such as $\mathrm{D} \alpha$, are most sensitive to Doppler broadening. If the density is sufficiently low, the Stark broadening is negligible and the spectral profile is proportional to the atomic VDF (see Fig. 1); this property has already been used for characterizing the recycling mechanisms of the neutrals present at the edge 4 . The situation is different for high density conditions (typically if $N_{e} \geq 10^{14} \mathrm{~cm}^{-3}$ ) because the Stark effect broadening increases to become comparable to the Doppler broadening. Figure 2 shows an example of adjustment of $\mathrm{D} \alpha$ obtained from a simulation accounting for the simultaneous action of the Stark and Doppler broadenings at $N_{e}=10^{14} \mathrm{~cm}^{-3}, T_{e}=T_{i}=1 \mathrm{eV}$, and assuming a Maxwellian distribution of atoms at $1 \mathrm{eV}$. The Zeeman splitting is not retained here for the sake of simplicity in discussions. In the adjustment model, only Doppler broadening is retained. The use of a Maxwellian distribution yields a systematic overestimate of the temperature due to the extended Stark line wing. A better agreement with a many-temperature Maxwellian model (e.g., to account for a population of hot neutrals created by charge exchange) is obtained but the physical interpretation of the temperatures is meaningless. The adjustments presented in figure 2 were performed with a genetic algorithm (see $[11$ for the code) applied using one- and two-temperature models. A value of $1.3 \mathrm{eV}$ was obtained from the one-temperature model and values of 1.6 and $16 \mathrm{eV}$ were obtained from the two-temperature model. So the conclusion is that a $\mathrm{D} \alpha$ line shape cannot always be fitted by just a Doppler broadened shape (with an adjusted VDF), as also Stark broadening can contribute significantly to the line shape for $N_{e}>10^{14} \mathrm{~cm}^{-3}$.

\section{An analysis of $\mathrm{D} \delta$ and $\mathrm{D} \varepsilon$ at high density conditions}

The relative contribution of Doppler broadening with respect to Stark broadening decreases as the principal quantum number $n$ increases (the dipole operator scales as $n^{2}$ and, hence, the Stark effect increases with $n$ ). In this framework, a line shape analysis using a pure Stark broadening model, i.e. not accounting for the Doppler effect, is appropriate if the principal quantum number $n$ is sufficiently high. Figure 3 shows a plot of the half-width at half-maximum (HWHM) of the first Balmer lines in terms of the principal quantum number for various densities. The HWHM is weakly sensitive to the ion and electron temperatures, hence we have assumed a value of $1 \mathrm{eV}$, which is representative of values measured in detached regime. The Doppler HWHM corresponding to Maxwellian distribution at $1 \mathrm{eV}$ is also shown in the figure. No values are displayed for $\mathrm{D} \beta(n=4)$ at $N_{e}=10^{14}$ and $10^{15} \mathrm{~cm}^{-3}$ as this line shows a 


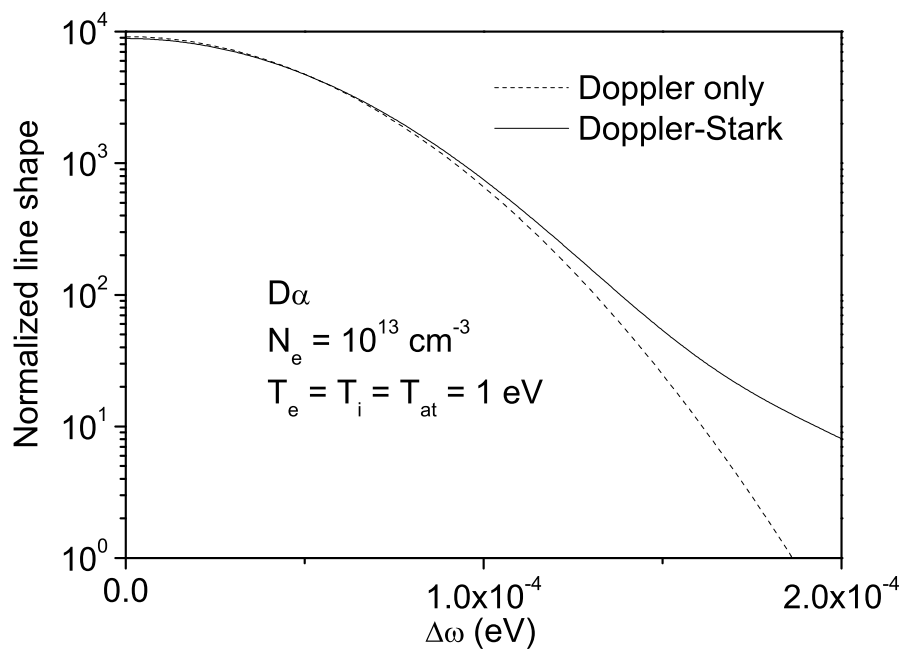

Figure 1: Plot of a calculated spectral profile of $\mathrm{D} \alpha$. At low densities, the contribution of the Stark broadening to the line width is negligible compared to that of the Doppler broadening in the case of lines with a low upper principal quantum number. It essentially provides a contribution to the line wing. Here, the spectrum is shown in semi-logarithmic scale in order to magnify the Stark effect contribution in the far wing.

dip at line center, rendering the concept of HWHM meaningless. The Stark effect contribution becomes larger than the Doppler broadening at $N_{e} \geq 10^{14} \mathrm{~cm}^{-3}$ for $n \geq 5$, indicating that a Stark broadening alone can be used safely in a diagnostic routine for the Balmer lines beyond $\mathrm{D} \beta$.

Figure 4 shows an example of a spectrum analysis. We have performed an adjustment of $\mathrm{D} \delta$ and $\mathrm{D} \varepsilon$ observed in the divertor of $\mathrm{TCV}$ in detached regime (shot \#52062 at $t=1.0 \mathrm{~s}$, central line of sight $[3]$ ). The real time application of the numerical simulation technique presented in Sec. 2 is not convenient so, in this framework, we have constructed a database directly exploitable by an adjustment program through an interpolation routine (see $[10$ ). The instrumental function was retained through convolution. Since the Stark broadening is weakly sensitive to temperature, we again assumed a value of $1 \mathrm{eV}$ with the density left as the only adjustable parameter. The best fit results are in a good agreement with the experimental spectra. Values of $5 \times 10^{13} \mathrm{~cm}^{-3}$ and $6 \times 10^{13} \mathrm{~cm}^{-3}$ were obtained for the electron density; the deviation between these values falls in the typical range of uncertainty expected from the accuracy of line shape model and experiment (in particular this includes the fitting method and the instrumental function characterization). Values of the same order of magnitude were obtained previously using a different Stark line shape model 3 . 


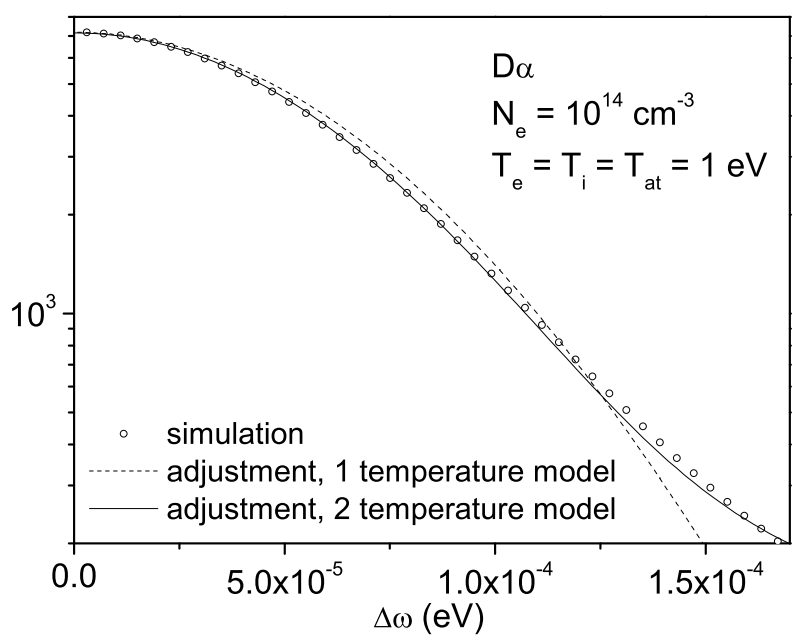

Figure 2: At high density conditions, the Stark effect on low $n$ lines can be comparable to the Doppler broadening contribution and spectra can be misinterpreted if this effect is not retained in diagnostic routines. Here, a calculated profile of $\mathrm{D} \alpha$ has been analyzed using a Doppler line broadening model not accounting for the Stark effect. The use of a Maxwellian distribution yields a systematic overestimate of the temperature ( $30 \%$ here) due to the inability of the model to reproduce the Stark line wing. If a two-temperature Maxwellian model is used instead, there is a better agreement between the curves but the physical interpretation is meaningless.

\section{Conclusion}

The operation of magnetic fusion devices requires passive spectroscopy diagnostics in the edge plasma region. In this work, we have applied a computer simulation technique to the modeling of Balmer line shapes in dense divertor conditions. Calculations in high-density conditions, assuming $1 \mathrm{eV}$ temperature and Gaussian atomic velocity distribution function, have indicated that the Stark effect contribution to line broadening becomes larger than the Doppler broadening at $N_{e} \geq 10^{14} \mathrm{~cm}^{-3}$ for $n \geq 5$, implying that Stark broadening alone can be used safely in a diagnostic routine for the Balmer series above $\mathrm{D} \beta$. This result was illustrated through an experimental spectrum analysis. An extension of this work to the modeling of Balmer lines in the presence of a strong magnetic field is currently in preparation for applications to the ITER divertor (B will be increased to $8 \mathrm{~T}$ ). Specific investigations accounting for the Zeeman effect will be performed. More sophisticated fitting algorithms devoted to cases where the Stark and Doppler contributions are comparable will also be investigated.

\section{Acknowledgments}

This work has been carried out within the framework of the EUROfusion Consortium and has received funding from the Euratom research and training programme 2014-2018 under grant agreement No 633053. The views and opinions expressed herein do not necessarily reflect those of the European Commission. 


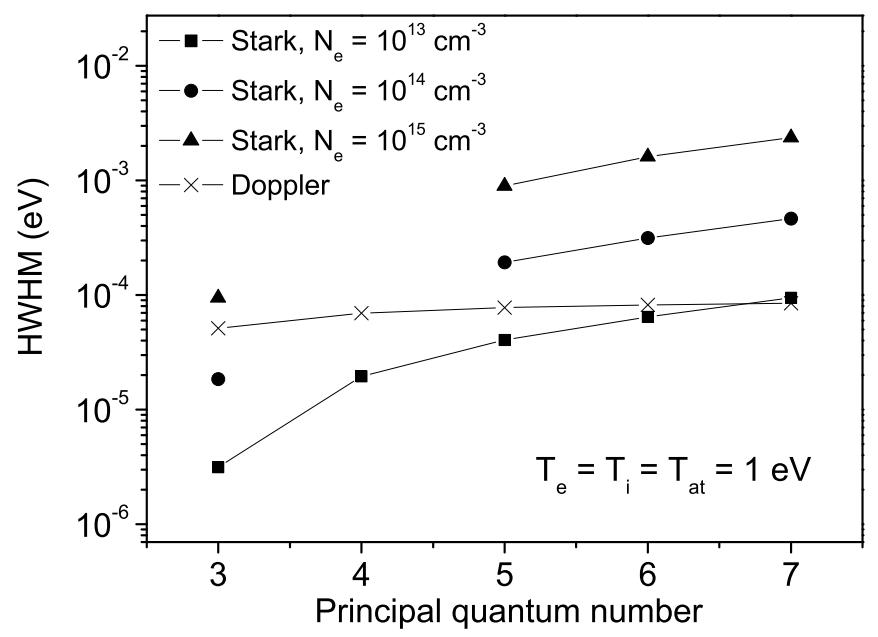

Figure 3: Plot of the half-width at half-maximum (HWHM) of the first Balmer lines in terms of the upper principal quantum number $n$ for various density values. A value of $1 \mathrm{eV}$ is assumed for the ion and electron temperatures. The Doppler HWHM corresponding to a $1 \mathrm{eV}$ temperature Maxwellian distribution is also shown. No values are displayed for $\mathrm{D} \beta(n=4)$ at $N_{e}=10^{14}$ and $10^{15} \mathrm{~cm}^{-3}$ because this line presents a dip at the center.
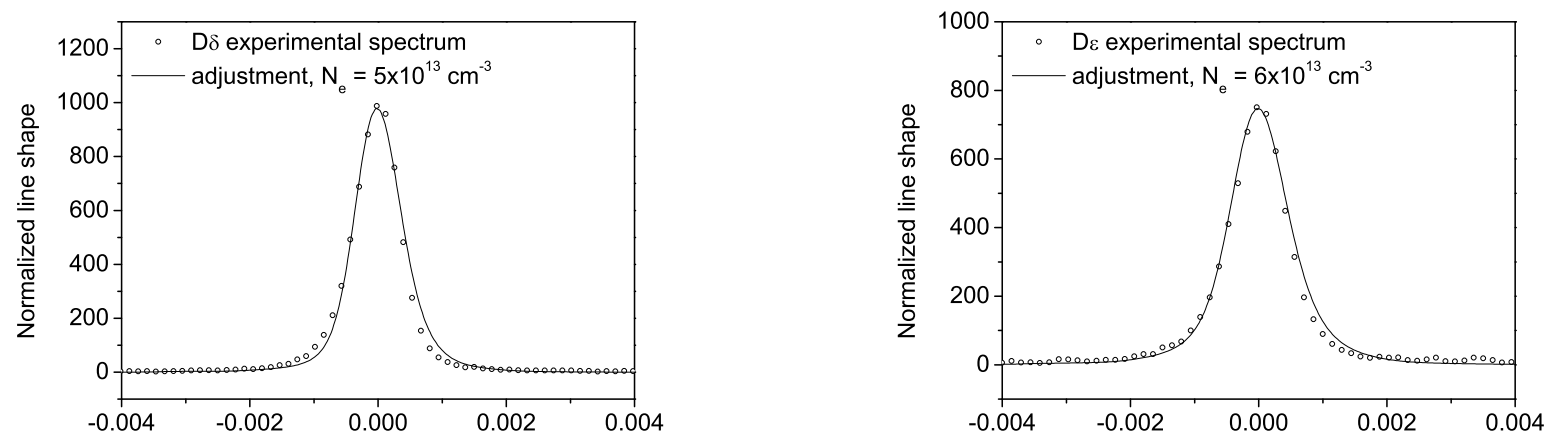

Figure 4: Plot of the spectral profiles of $\mathrm{D} \delta$ and $\mathrm{D} \varepsilon$, obtained experimentally (circles) and from a calculation (solid line). An adjustment has been done using the Stark broadening model presented in this work. Values of $5 \times 10^{13}$ $\mathrm{cm}^{-3}$ and $6 \times 10^{13} \mathrm{~cm}^{-3}$ have been obtained for the electron density. The deviation between these values falls in the typical range of uncertainty provided by the model accuracy.

\section{References}

[1] S. Potzel et al., Plasma Phys. Control. Fusion 56, 025010 (2014).

[2] B. A. Lomanowski et al., Nucl. Fusion 55, 123028 (2015).

[3] K. Verhaegh et al., Nucl. Mater. Energy 12, 1112 (2017).

[4] J. D. Hey, C. C. Chu, and E. Hintz, J. Phys. B: At. Mol. Opt. Phys. 32, 3555 (1999). 
[5] M. Koubiti et al., J. Quant. Spectrosc. Radiat. Transfer 71, 455 (2001).

[6] J. Rosato et al., Phys. Rev. E 79, 046408 (2009).

[7] H. R. Griem, Spectral Line Broadening by Plasmas (Academic Press, London, 1974).

[8] R. Stamm and D. Voslamber, J. Quant. Spectrosc. Radiat. Transfer 22, 599 (1979).

[9] E. Stambulchik and Y. Maron, High Energy Density Phys 6, 9 (2010).

[10] J. Rosato, Y. Marandet, and R. Stamm, J. Quant. Spectrosc. Radiat. Transfer 187, 333 (2017).

[11] P. Charbonneau, Astrophys. J. Suppl. Ser. 101, 309 (1995). 
REFERENCES

$\square$ 